TITLE:

\title{
Evaluation index of acceleration factor and ordering in shifted ICCG method for electromagnetic field analyses
}

\section{$\operatorname{AUTHOR}(S)$ :}

Iwashita, Takeshi; Mifune, Takeshi; Shimasaki, Masaaki

\section{CITATION:}

Iwashita, Takeshi ... [et al]. Evaluation index of acceleration factor and ordering in shifted ICCG method for electromagnetic field analyses. IEEE TRANSACTIONS ON MAGNETICS 2007, 43(4): 1493-1496

ISSUE DATE:

2007-04

URL:

http://hdl.handle.net/2433/50513

\section{RIGHT:}

(c)2007 IEEE. Personal use of this material is permitted. However, permission to reprint/republish this material for advertising or promotional purposes or for creating new collective works for resale or redistribution to servers or lists, or to reuse any copyrighted component of this work in other works must be obtained from the IEEE. 


\title{
Evaluation Index of Acceleration Factor and Ordering in Shifted ICCG Method for Electromagnetic Field Analyses
}

\author{
Takeshi Iwashita $^{1}$, Takeshi Mifune ${ }^{2}$, and Masaaki Shimasaki ${ }^{2}$ \\ ${ }^{1}$ Academic Center for Computing and Media Studies, Kyoto University, Kyoto, 606-8501 Japan \\ ${ }^{2}$ Graduate School of Engineering, Kyoto University, Kyoto, 615-8510 Japan
}

\begin{abstract}
This paper introduces a simple way to evaluate the preconditioning effect in the shifted ICCG method. The proposed evaluation index is easily calculated with little additional memory in an incomplete factorization process; thus, it can be used for setting appropriate parameters for the solver, such as an acceleration factor and the ordering of unknowns, prior to the time-consuming iteration process. Numerical results show that the evaluation index gives the good estimate of the number of iterations necessary for convergence.
\end{abstract}

Index Terms-Convergence estimation, evaluation index, finite-edge element analysis, ordering, shifted ICCG method.

\section{INTRODUCTION}

$\mathbf{T}$ HE solution process in a linear system of equations is the most time consuming part in a finite-element analysis of electromagnetic field problems. While a multigrid or multilevel method has recently been developed for the solution step, the incomplete Choleskey conjugate gradient (ICCG) method [1] remains one of the most popular linear solvers in electromagnetic field analyses. In an electromagnetic field analysis based on the finite-edge element method, A- or A-V methods are often used [2], [3]. In these methods, the global coefficient matrix is semipositive; therefore, the normal IC preconditioning cannot be directly applied to the coefficient matrix because of a breakdown in the incomplete factorization process. A popular remedy for this is to construct a preconditioning matrix through incomplete factorization of a positive definite matrix approximate to the original coefficient matrix [4]. The shifted IC preconditioning (the shifted ICCG method) is one such remedy and is widely used in electromagnetic field analyses [5].

In the shifted ICCG method, the convergence is greatly affected by the acceleration factor that determines the shift quantity and the ordering of unknowns [6]. Furthermore, when parallel orderings are used for an analysis performed in a parallel computation environment, engineers have to be careful about more parameters that can affect the convergence. In this paper, we present a convergence evaluation index as a tool to assist engineers to select appropriate parameters or orderings. The evaluation index can be easily calculated prior to an iterative solution process. The authors recently proposed an easy way to evaluate convergence in ILU preconditioning in structured analyses [7], and in this paper, we further develop the method to include unstructured analyses. The proposed evaluation index Precise

Digital Object Identifier 10.1109/TMAG.2007.892472
Remainder Index (P.R.I.) can be easily computed with little memory requirement. Numerical tests confirm the effectiveness of the proposed method in a finite-edge element electromagnetic field analysis.

\section{ILU PRECONDITIONING}

This paper deals with the following $n$-dimensional linear system of equations:

$$
A \boldsymbol{u}=\boldsymbol{f}
$$

While the coefficient matrix is symmetric and semi-positive definite, our evaluation method is explained in a general format and includes a nonsymmetric coefficient matrix case.

When the linear system of equations is solved by iterative methods, preconditioning techniques are often used [8]. In this technique, the linear system is transformed into a preconditioned system

$$
\left(\boldsymbol{K}_{1}^{-1} A \boldsymbol{K}_{2}^{-1}\right)\left(\boldsymbol{K}_{2} \boldsymbol{u}\right)=\boldsymbol{K}_{1}^{-1} \boldsymbol{f}
$$

that accelerates the convergence of a basic iterative method. The matrix $M=K_{1} K_{2}$ is called a preconditioner matrix. In ILU preconditioning, the preconditioning matrix is given by

$$
\boldsymbol{M}=\boldsymbol{L} \boldsymbol{D}^{-1} \boldsymbol{U}
$$

where $\boldsymbol{L}, \boldsymbol{D}$, and $\boldsymbol{U}$ are a lower triangular matrix, a diagonal matrix, and an upper triangular matrix, respectively. These matrices $L, D$, and $\boldsymbol{U}$ are derived from ILU factorization of the coefficient matrix $\boldsymbol{A}$ as follows:

$$
\boldsymbol{A}=\left(\boldsymbol{L} \boldsymbol{D}^{-1} \boldsymbol{U}\right)-\boldsymbol{R}_{\mathrm{ilu}}
$$

where $\boldsymbol{R}_{\text {ilu }}$ is the matrix of the elements that are dropped during the incomplete factorization. 
In the ILU preconditioned iterative method algorithm, the transformation (2) is not performed explicitly, and the preconditioning step is given by the solution of a linear system

$$
\left(\boldsymbol{L} \boldsymbol{D}^{-1} \boldsymbol{U}\right) \boldsymbol{z}=\boldsymbol{x} .
$$

Since the preconditioner matrices depend on the ordering of the unknowns, the preconditioning effect is also affected by the ordering. Moreover, the degree of parallelism in the solution (5) depends on the ordering.

\section{New EVAluation INDEX OF CONVERGENCE}

\section{A. Remainder Matrix}

A typical way to evaluate the preconditioning effect is through checking the condition number or the eigenvalues of the preconditioned coefficient matrix. However, as the computational cost of computing eigenvalues is generally high, the following method proposed by Duff and Meurant is widely used for evaluating the preconditioning effect.

Duff and Meurant's method [9].

The effect of ILU preconditioning, is evaluated by the norm of the remainder matrix $\boldsymbol{R}$

$$
\boldsymbol{R}=\boldsymbol{M}-\boldsymbol{A} .
$$

A smaller norm of $\boldsymbol{R}$ results in better convergence.

In the ILU preconditioning case, it holds that

$$
R=R_{\text {ilu }} .
$$

Duff and Meurant used the Frobenius norm of the remainder matrix and their numerical tests of finite-difference analyses confirmed the effectiveness of their method [9]. However, when the ILU preconditioning technique is applied to a general sparse coefficient matrix, the additional memory requirement and computational cost for computing the Frobenius norm of the remainder matrix are not small compared with the iterative solution process. Therefore, another practical method is required for evaluating preconditioning effects in unstructured analyses.

\section{B. P.R.I.}

In this section, we describe a new evaluation index, which is called P.R.I. [7]. To construct a new evaluation index, we consider the remainder matrix in ILU preconditioning. The remainder matrix $R$ can be computed by using the algorithm associated with ILU factorization as shown in Fig. 1. When we focus on an arbitrary element $r_{J, K}$ in the algorithm, the element is updated several times depending on the non-zero element pattern of the coefficient matrix. Since these update quantities are different from each other, storing all dropped fill-ins is necessary for computing the exact remainder matrix entries. Thus, in the P.R.I. evaluation, we use a summation of the absolute values of the updates of the remainder matrix. The algorithm for calculating the P.R.I. value $I_{\mathrm{rp}}$ is shown in Fig. 1. The additional memory requirement for the calculation is for only one variable. Moreover, the computational cost is generally much

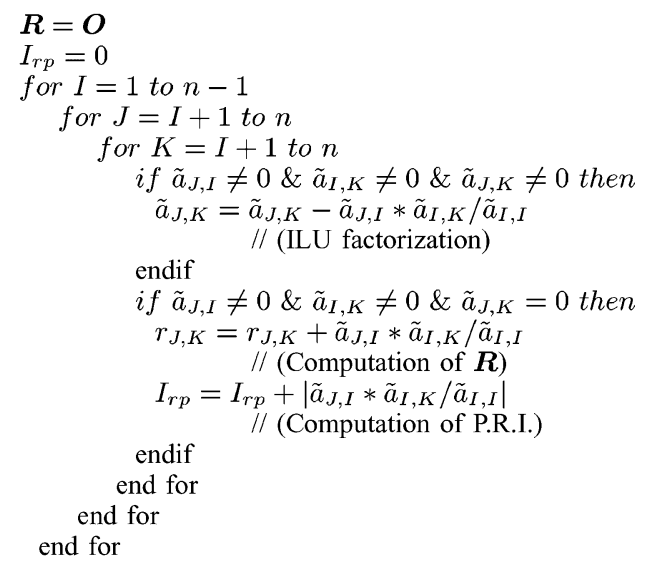

Fig. 1. Algorithm for ILU factorization with computing of the remainder matrix and the P.R.I.

smaller than the iteration process. When all diagonal entries of $\boldsymbol{A}$ have the same signs and all off-diagonal entries of $\boldsymbol{A}$ also have the identical signs, a simple relationship between the remainder matrix norm and the P.R.I. is obtained as follows:

$$
\|\boldsymbol{R}\|_{A}=I_{\mathrm{rp}}
$$

where $\|\boldsymbol{R}\|_{A}$ is defined as a sum of the absolute values of all entries of $\boldsymbol{R}$, and is given by

$$
\|\boldsymbol{R}\|_{A}=\sum_{I J}\left|r_{I J}\right|
$$

The operator $\|\cdot\|_{A}$ satisfies the definition of the matrix norm shown in [10]. For example, the relationship (8) is satisfied in a finite-difference analysis of a Poisson equation. In a general coefficient matrix case, since the dropped fill-ins may have different signs

$$
\|R\|_{A} \leq I_{\mathrm{rp}}
$$

is obtained. Moreover, the Frobenius norm of the remainder matrix $\|\boldsymbol{R}\|_{F}$ and $\|\boldsymbol{R}\|_{A}$ have the following relationship:

$$
\|\boldsymbol{R}\|_{F}^{2}=\sum_{j, k}\left(r_{j, k}\right)^{2} \leq\left(\sum_{j, k}\left|r_{j, k}\right|\right)^{2}=\|\boldsymbol{R}\|_{A}^{2} .
$$

Consequently, the P.R.I. gives an upper bound of the Frobenius norm of the remainder matrix as follows:

$$
\|R\|_{F} \leq I_{\mathrm{rp}}
$$

\section{P.R.I. for Variants of ILU Preconditioning}

In practical analyses, ILU factorization can fail due to pivot breakdown. One remedy for such a breakdown is the modification of the coefficient matrix before factorization; for example, diagonal sifts [5], [11]. Let $\boldsymbol{A}_{\Delta m}$ be a modification term, then the modified factorization is given by

$$
\boldsymbol{A}+\boldsymbol{A}_{\Delta m}=\left(\boldsymbol{L}_{m} \boldsymbol{D}_{m}^{-1} \boldsymbol{U}_{m}\right)-\boldsymbol{R}_{\text {ilum }} .
$$


TABLE I

DISCRETIZATION DATA

\begin{tabular}{|c|c|}
\hline Number of volume elements & 327680 \\
\hline Number of nodes & 342225 \\
\hline Number of unknowns & 1011920 \\
\hline Time step & $1 \mathrm{msec}$ \\
\hline
\end{tabular}

For this factorization, the remainder matrix is written as follows:

$$
\boldsymbol{R}=\boldsymbol{R}_{\text {ilum }}+\boldsymbol{A}_{\Delta m} .
$$

Here, we define the P.R.I. value for the factorization (13) as

$$
I_{\mathrm{rpm}}=I_{r p 0}+\left\|\boldsymbol{A}_{\Delta m}\right\|_{A} .
$$

In (15), $I_{r p 0}$ is the $I_{\mathrm{rp}}$ value calculated in the algorithm shown in Fig. 1.

In the shifted ICCG method, the modification term is given as follows:

$$
\boldsymbol{A}_{\Delta m}=\operatorname{diag}(\boldsymbol{A}) \cdot \alpha
$$

where $\operatorname{diag}()$ means the diagonal part of a matrix and $\alpha$ is a positive scalar value, which in electromagnetic field analyses is generally called an acceleration factor.

\section{RESULTS}

\section{A. Test Model}

In the present analysis, we use the IEEJ standard benchmark model of 3-D eddy-current analyses [2]. The analyzed model is discretized by first-order brick-type edge elements. Table I lists the discretization data. The electromagnetic field equations are solved by using the Galerkin method with $A$-formulation and the backward time difference method. The linear system of equations generated is solved by the shifted ICCG method. The convergence criterion of the iterative method is given by $\|\boldsymbol{r}\|_{2} /\|\boldsymbol{f}\|_{2}<10^{-7}$, where $\boldsymbol{r}$ and $\boldsymbol{f}$ are the residual vector and the right-hand-side vector of the linear system, respectively.

\section{B. Numerical Results}

In this paper, we examined effects of the acceleration factor $\alpha$ and orderings on the convergence rate in the shifted ICCG method. While there are several orderings for a finite-element analysis, we pay special attention to multicolor ordering, which is one of the most popular ways for parallelization in the ICCG method. It is well known that parallel orderings including multicolor ordering suffer from a tradeoff problem between parallelism and convergence of the iterative solver [12].

Fig. 2 shows the convergence behavior of the shifted ICCG method when the unknown variables are ordered by using natural, random, 80-color and 400-color orderings. In this numerical test, the acceleration factor is fixed at 1.08. Fig. 2 indicates that ordering of the unknowns affects the convergence rate of the iterative solver. Although the relationship between ordering and convergence is generally not simple in unstructured analyses, the result shown in Fig. 2 agrees with previous research results obtained in structured finite-difference analyses [9], [12]. While

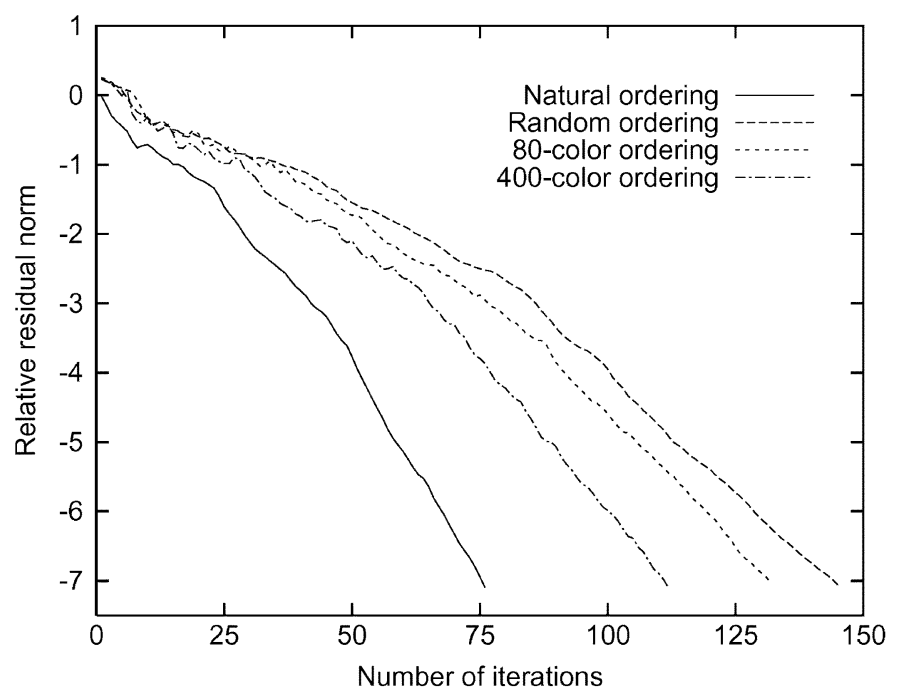

Fig. 2. Comparison of the convergence rate of the orderings.

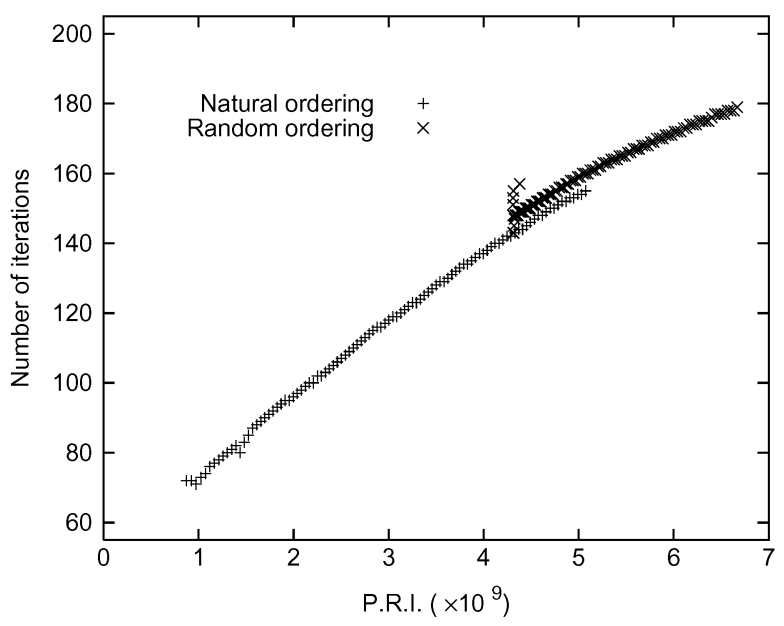

Fig. 3. Relationship between the P.R.I. and the number of iterations.

natural ordering shows the best preconditioning performance, the increased number of colors in multicolor ordering improves the convergence rate.

Next, we examine the effectiveness of the P.R.I. on the estimation of the number of iterations needed to obtain convergence in the iterative solver. Fig. 3 plots the relationship between the P.R.I. and the number of iteration in natural and random orderings when the acceleration factor is changed from 1.03 to 2.03 . In Fig. 3, the numerical tests based on two different orderings form an identical P.R.I. evaluation line. This result indicates that the P.R.I. is effective for comparisons between different orderings in the shifted ICCG method even when the acceleration factor varies. Figs. 4 and 5 show the results of multicolor ordering with various numbers of colors where the acceleration factor is set to be $1.08,1.16$, or 1.2. In Fig. 4, we observe a general characteristic of multicolor ordering, in which an increase in the number of colors reduces the number of iterations. However, the relationship between the number of colors and the number of iteration is not given by a simple linear function. On 


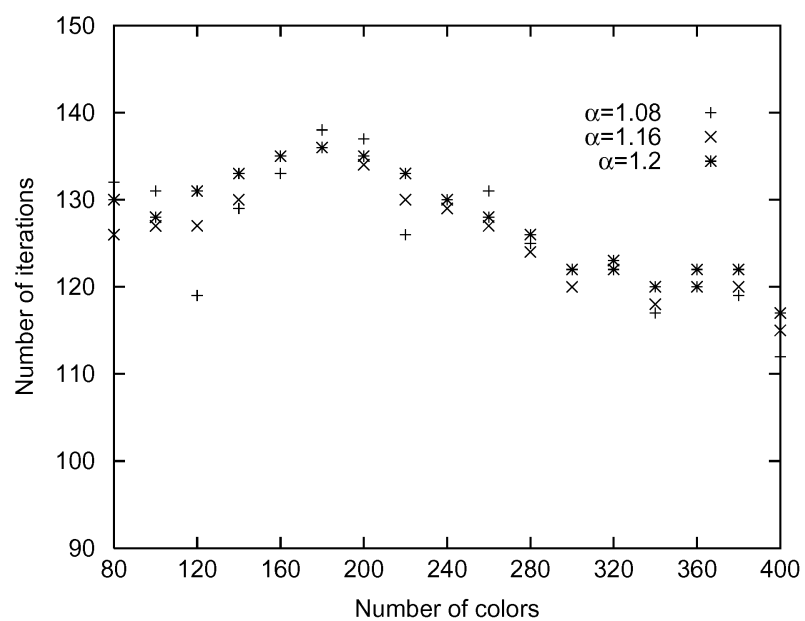

Fig. 4. Relationship between the number of colors and the number of iterations in the multicolor ordering technique.

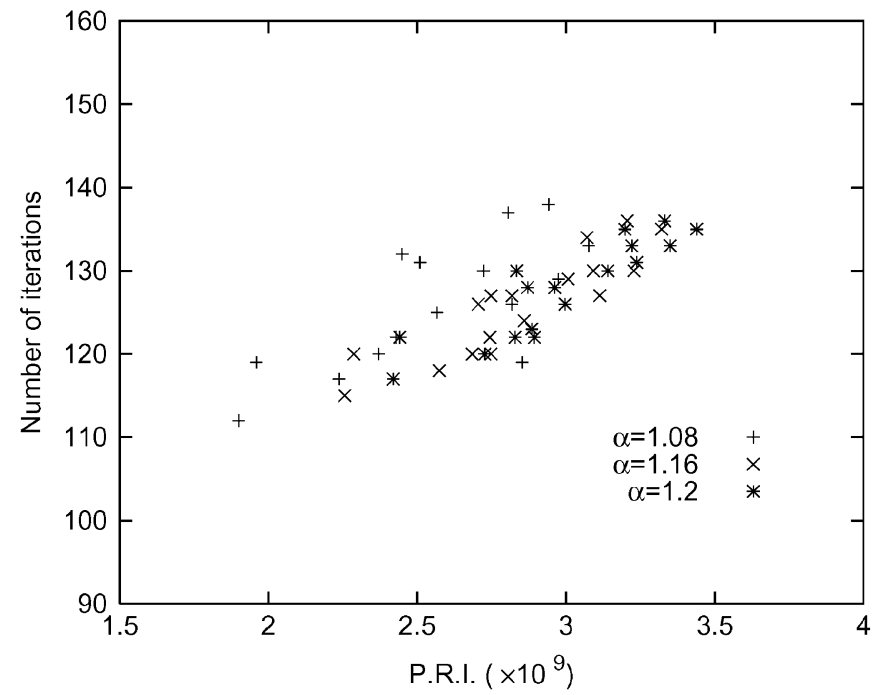

Fig. 5. Relationship between the P.R.I. and the number of iterations in the multicolor ordering technique.

the other hand, in the same numerical test, the number of iterations is roughly proportional to the P.R.I. as shown in Fig. 5. Since the degree of parallelism is in an inverse proportion to the number of colors, the P.R.I. value can be used for setting the number of colors to compromise parallelism and convergence in the multicolor ordering technique.

Next, we check a comparison of convergence evaluation accuracies between the P.R.I. and the Frobenius norm of the remainder matrix $\left\|\boldsymbol{R}_{F}\right\|$ that is a conventional convergence evaluation index [9]. Fig. 6 shows a strong correlation between two evaluation indices. Namely, the P.R.I. has the same degree of convergence evaluation accuracy as the $\left\|\boldsymbol{R}_{F}\right\|$ value. On the other hand, computing the $\left\|\boldsymbol{R}_{F}\right\|$ value requires additional memory, which is twice as large as the memory requirement for the coefficient matrix. Accordingly, the P.R.I. has the advantage of a much less memory requirement.

\section{CONCLUSION}

This paper introduces the convergence evaluation index (P.R.I.) in the shifted ICCG method. The evaluation index is

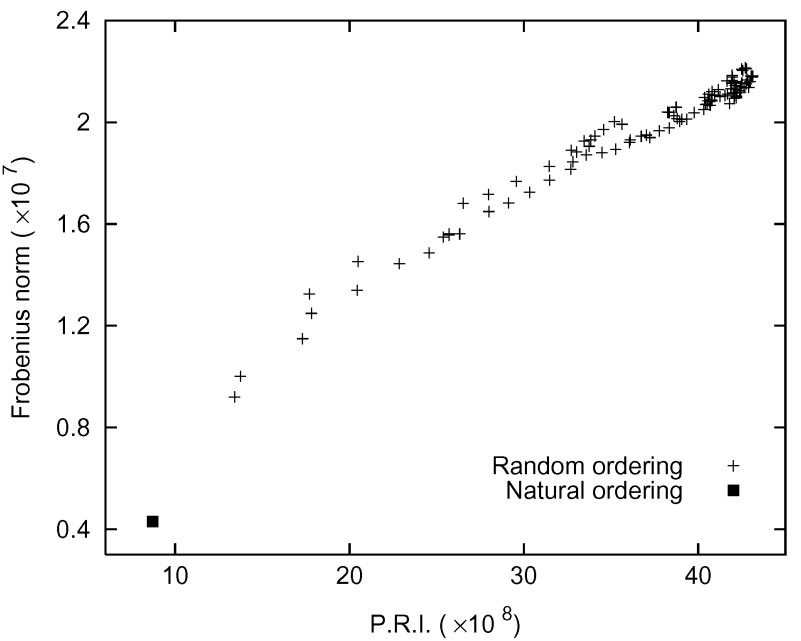

Fig. 6. Relationship between the P.R.I. and the Frobenius norm of the remainder matrix.

easily computed with little additional memory. Numerical tests confirm the relationship between the number of iterations and the P.R.I. values when different orderings and acceleration factors are used. Moreover, it is shown that the P.R.I. has a strong correlation with the Frobenius norm of the remainder matrix that is a conventional evaluation index. The memory requirement for computing the P.R.I. value is much less than that for the conventional index. Consequently, P.R.I. values can be used as a tool for estimating the number of iterations in setting parameters in the shifted ICCG method.

\section{REFERENCES}

[1] J. Meijerink and H. A. van der Vorst, "An iterative solution method for linear systems of which the coefficient matrix is a symmetric M-matrix," Math. Comput., vol. 31, pp. 148-162, 1977.

[2] T. Nakata, N. Takahashi, T. Imai, and K. Muramatsu, "Comparison of various methods of analysis and finite elements in 3-D magnetic field analysis," IEEE Trans. Magn., vol. 27, no. 5, pp. 4073-4076, Sep. 1991.

[3] K. Fujiwara, T. Nakata, and H. Ohashi, "Improvement of convergence characteristic of ICCG method for the $A-\phi$ method using edge elements," IEEE Trans. Magn., vol. 32, no. 3, pp. 804-807, May 1996.

[4] I. Hladik, M. B. Reed, and G. Swoboda, "Robust preconditioners for linear elasticity FEM analyses," Int. J. Numerical Methods Eng., vol. 40, pp. 2109-2127, 1997.

[5] T. A. Manteuffel, "An incomplete factorization technique for positive definite linear systems," Math. Comput., vol. 34, pp. 473-497, 1980.

[6] K. Fujiwara, T. Nakata, and H. Fusayasu, "Acceleration of convergence characteristic of the ICCG method," IEEE Trans. Magn., vol. 27, no. 2, pp. 1958-1961, Mar. 1993.

[7] T. Iwashita, Y. Nakanishi, and M. Shimasaki, "Comparison criteria for parallel orderings in ILU preconditioning," SIAM J. Sci. Comput., vol. 26, no. 4, pp. 1234-1260, 2005.

[8] Y. Saad, Iterative Methods for Sparse Linear Systems, 2nd ed. Philadelphia, PA: SIAM, 2003.

[9] I. S. Duff and G. A. Meurant, "The effect of ordering on preconditioned conjugate gradients," BIT, vol. 29, pp. 635-657, 1989.

[10] J. Demmel, Applied Numerical Linear Algebra. Philadelphia, PA: SIAM, 1997.

[11] M. Benzi and M. Tuma, "A robust incomplete factorization preconditioner for positive definite matrices," Numer. Linear Algebra Appl., vol. 10, pp. 385-400, 2003.

[12] S. Doi and T. Washio, "Ordering strategies and related techniques to overcome the trade-off between parallelism and convergence in incomplete factorization," Parallel Comput., vol. 25, pp. 1995-2014, 1999.

Manuscript received April 24, 2006 (e-mail: iwashita@media.kyoto-u.ac.jp). 